\title{
Title: Targeting of Organophosphorus Compound Bioscavengers to the Surface of Red Blood Cells
}

Authors: Bryan J. McCranor ${ }^{1}$, Catherine A. Hofstetter, Melissa A. Olert, Deborah MooradDoctor, Douglas M. Cerasoli, Gregory E. Garcia

\section{Abstract}

To develop a prophylactic for organophosphorus (OP) poisoning utilizing catalytic bioscavengers, the circulatory stability of the enzymes needs to be increased. One strategy for increasing the bioavailability of OP bioscavengers is to target them to the surface of red blood cells (RBCs). Given the circulatory lifespan of 120 days for human RBCs, this strategy has the potential for creating a persistent pool of bioscavenger. Here we report the development of fusion proteins with a single chain variable fragment (scFv) of Ter119, a molecule that associates with glycophorin A on the surface of RBCs, and the VIID11 variant of paraoxonase 1 (scFv-PON1). We show that scFv-PON1 variants expressed by Trichoplusia ni larvae are catalytically active and that one variant in particular can successfully bind to the surface of murine RBCs both in vitro and in vivo. This study represents a proof of concept for targeting catalytic bioscavengers to the surface of RBCs and is an early step in developing catalytic bioscavengers that can remain in circulation for an extended period of time.

\section{Introduction}

Organophosphorus compounds (OP) are highly poisonous chemicals that prevent normal function of the nervous system through the irreversible binding and inactivation of acetylcholinesterase (AChE) [1, 2]. Inhibition of AChE leads to a buildup of acetylcholine in the central and peripheral nervous system, resulting in a cholinergic syndrome consisting of respiratory and oral secretions, diarrhea and vomiting, sweating, altered mental status, autonomic instability, general weakness, and progression to paralysis and respiratory arrest [2, 3]. In the United States the standard of care for OP poisoning is treatment with atropine, which reduces binding of acetylcholine to muscarinic receptors, and pyridinium-2-aldoxime (2-PAM $\mathrm{Cl})$, which can reactivate inhibited AChE [2-5]. If administered shortly after intoxication, these medical countermeasures are successful in preventing death, but do not alleviate performance deficits in the warfighter caused by the symptoms of OP poisoning $[4,6]$. This, in addition to the 
side-effects from the therapeutics themselves, can leave units temporarily depleted and vulnerable to future attack. The issue of how to treat OP exposures with a minimum of physiological and cognitive consequences has led to the concept and development of OP bioscavengers [4, 7-12]. Desirable characteristics for prophylactic bioscavengers include easy administration (i.e., intramuscular rather than intravenous injection), broad specificity, lack of physiological, cognitive, behavioral, or immunological effects, and sustained stability in the circulation. One strategy for designing a catalytic bioscavenger is based on paraoxonase 1 (PON1), which has been the subject of a concerted, directed evolution effort to improve its activity vs. G-agents [13]. PON1 variants have been identified with sufficient activity to be candidates for further development, and new technologies are needed to improve the in vivo retention of this protein for effective prophylactic use.

A strategy for prolonging the bioavailability of OP bioscavengers is to sequester them onto red blood cells (RBCs), improving their retention and stability. Approximately $30 \times 10^{12}$ RBCs at any given time are circulating in the human body [14], providing great potential for surface labeling, distribution, and retention in the vascular compartment. Additionally, human RBCs have an average circulatory lifetime of 120 days [15]. Use of a RBC-specific membrane protein such as glycophorin A (GPA), the major cell surface sialoglycoprotein which accounts for $1.6 \%$ of the total $\mathrm{RBC}$ membrane protein, equaling about $10^{6}$ copies/cell [16-18], as a target with which to associate an enzyme could theoretically yield an in vivo $\mathrm{C}_{\max }$ of $40 \mu \mathrm{M}$ and create a bioscavenger depot of fully accessible and reactive enzyme. This technique has been previously utilized successfully in rodent models to prolong the in vivo retention of blood coagulation therapeutic proteins such as decay accelerator factor (DAF) and tissue plasminogen activation factor (tPA). The in vivo results were compelling as RBC fusion dramatically enhanced recombinant protein retention times. DAF exhibited a loss of $10-12 \%$ per day [19], while over $80 \%$ of tPA activity was retained after 2 days [20]. The decay rates of these fusion proteins parallel the circulatory half-life of mouse RBCs [21].

Here we describe work to develop an OP bioscavenger with a GPA-associating single chain variable fragment (scFv) based on the Ter119 antibody to increase the bioavailability of PON1 by targeting the bioscavenger to RBCs. The Ter119 antibody recognizes the antigen Ter119, a $52 \mathrm{kDa}$ molecule on the surface of murine RBCs that is known to highly associate with GPA [22]. We observed no significant impact on PON1 activity from fusion with the scFv, and we observed the successful binding of a scFv-PON1 variant to murine RBCs both in vivo and in vitro. Proteins delivered in this manner could theoretically improve the retention times to 
as long as those of the native RBC (approximately 120 days). With such treatment it would be possible to retain effective concentrations of the proteins over a time course relevant to warfighters and first responders and to reduce or eliminate the need for multiple dosing to maintain protective levels of enzyme in the circulation.

\section{Materials and Methods}

Fusion Proteins: Fusion proteins of a scFv for Ter119 [19] and the VIID11 [23, 24] variant of PON1 were purchased from Chesapeake PERL Inc. (Savage, MD). The enzymes were expressed by Chesapeake PERL using their Trichoplusia ni larval expression system [25]. Variants were constructed according to the strategy in Figure 1. A GSSSGSSS linker was used between scFv and PON1 sequences, and a $\mathrm{C}$ terminal His6x tag was included in the fusion protein to assist with isolation and purification [26].

SDS-PAGE of fusion proteins: Protein samples were prepared for SDS-PAGE with 2X Laemmli Sample Buffer (Bio-Rad, Hercules, CA) and 2-Mercaptoethanol (Sigma-Aldrich, St. Louis, MO) according to the manufacturer's protocol. Gels were run using Mini-Protean TGX gels (Bio-Rad, Hercules, CA) in Tris/Glycine/SDS Buffer (Bio-Rad, Hercules, CA) and Mark 12 unstained standard (Life Technologies, Carlsbad, CA). Gels were run at 100-130V and then stained with eStain2.0 and protein staining pads (GenScript, Piscataway, NJ) according to the manufacturer's protocol. Gels were then imaged on an Odyssey CLx (LiCor Biosciences, Lincoln, NE) using Image Studio software.

In vitro PON1 activity: In vitro enzyme activity was assessed using a spectrometric paraoxonase activity assay [27]. The assay was run in a 96-well plate format with a final reaction volume of $200 \mu \mathrm{l}$ per well. PON1 fusion proteins were added at a volume of $10 \mu \mathrm{l}$ to the plates, along with $180 \mu \mathrm{l}$ of assay buffer consisting of $50 \mathrm{mM}$ HEPES pH 7.2 and $10 \mathrm{mM}$ $\mathrm{CaCl}_{2}$ (Sigma-Aldrich, St. Louis, $\mathrm{MO}$ ). Increasing concentrations of paraoxon (Chem Service Inc., West Chester, PA) were added at volumes of $10 \mu$ to each well. Immediately following the addition of paraoxon the plate was read using a SpectraMax Plus 384 (Molecular Devices, Sunnyvale, CA) in kinetic mode at a wavelength of $412 \mathrm{~nm}$. Following a $30 \mathrm{sec}$ plate shake, the plate was read in $20 \mathrm{sec}$ intervals for $10 \mathrm{~min}$. The velocity of the reaction in milli-absorbance units per minute ( $\mathrm{mAU} / \mathrm{min}$ ) was calculated by the instrument's software. Using the BeerLambert Law and the extinction coefficient of $1.8 \times 10^{4} \mathrm{M}^{-1} \mathrm{~cm}^{-1}$, the reaction rate was converted 
from $\mathrm{mAU} / \mathrm{min}$ to $\mathrm{M} / \mathrm{min}$ and plotted vs. paraoxon concentration. $\mathrm{V}_{\max }$ and $\mathrm{k}_{\mathrm{m}}$ were calculated from the plot using the Michaelis-Menton model with GraphPad Prism 5 software (GraphPad Software Inc., La Jolla, CA), and $k_{\text {cat }}$ and the catalytic efficiency $\left(k_{c a t} / k_{m}\right)$ were calculated from those values.

In vitro $R B C$ targeting: Approximately $25 \mu$ of mouse whole blood was obtained from male C57BL/6 mice aged 2-4 months, washed with PBS, 0.2\% BSA, and centrifuged for 5 min at 200 $x \mathrm{~g}$ at $4^{\circ} \mathrm{C}$. The RBC pellet was resuspended in $100 \mu \mathrm{PBS} \mathrm{pH} 7.4,0.2 \% \mathrm{BSA}$ and incubated with increasing concentrations of Ter119 scFv-PON1 fusion proteins (30, 45, and $75 \mu \mathrm{g} / \mathrm{ml}$ ) for $45 \mathrm{~min}$ on ice. Cells were centrifuged for $5 \mathrm{~min}$ at $200 \mathrm{xg}$ at $4^{\circ} \mathrm{C}$ and resuspended in $100 \mu \mathrm{l}$ PBS pH 7.4, 0.2\% BSA. 6xHis Epitope Tag Antibody, DyLight 680 conjugate (Thermo Scientific, Rockford, IL) was added to the cells at 1:100 and incubated for $30 \mathrm{~min}$ on ice. Cells were washed with PBS pH 7.4, 0.2\% BSA, centrifuged for $5 \mathrm{~min}$ at $200 \mathrm{xg}$ at $4^{\circ} \mathrm{C}$, and resuspended in $300 \mu \mathrm{PBS}$ pH 7.4, 0.2\% BSA. RBCs were analyzed by flow cytometry using a guava easyCyte ${ }^{\mathrm{TM}}$ flow cytometer (EMD Millipore, Billerica, MA), gating on live cells (as determined by forward and side scattering).

In vivo experiments: C57BL/6 background mice, age 2-6 months, were dosed with $5 \mathrm{mg} / \mathrm{kg}$ of fusion enzyme via intraperitoneal (IP) injection. Approximately $25 \mu \mathrm{l}$ of blood was collected at 0 , 2,24 , and $50 \mathrm{~h}$ via tail snip in a heparin blood collection tube. RBCs were separated from plasma via centrifugation at $10,000 \times \mathrm{g}$ for 10 mins at $4^{\circ} \mathrm{C}$. Plasma was removed and frozen at $80^{\circ} \mathrm{C}$ for further analysis. Cells were then analyzed by flow cytometry as stated above.

Plasma PON1 activity assay: Plasma PON1 activity of the mice was determined using similar methods to the in vitro assay described above. Frozen plasma was thawed, and $10 \mu \mathrm{l}$ of plasma and $0.035 \mathrm{M}$ paraoxon were used in the same assay conditions as the in vitro assay. The PON1 activity for each mouse at each time point was normalized to its baseline (activity in plasma samples collected at time point 0 ).

Data analysis: Statistical significance was determined for the in vitro data by using a Student's T-test with appropriate Bonferroni corrections and a significance threshold of 0.017. A twofactor ANOVA with repeated measures was used to compare the in vivo data's three treatment groups and three observation times. A significant treatment group by time interaction $(p<$ 
0.0001) was observed, indicating that the differences among treatment groups varied with time. Therefore, a Bonferroni post-test was used to compare pairs of treatments at each time point.

\section{Results}

Ter119 scFv-PON1 catalytic efficiency

We obtained Ter119 scFv-PON1 (VIID11) fusion protein variants from Chesapeake PERL, with an added 6xHis residue to aid in isolation and purification [26], which produced them using a Trichoplusia ni larval expression system [25] (Figure 1.). Samples were characterized by SDS-PAGE gel to confirm molecular weights correlated with each construct. As expected, the scFv-PON1 proteins (Figure 1) (AB771: PON1-scFv-His, AB772: scFv-PON1-His) and unconjugated control (PON1-His) ran at the predicted molecular weights (scFv-PON1 - 64 KDa, PON1 - $38 \mathrm{KDa}$ ) (Figure 2.). To confirm that the PON1 fusion proteins still retained enzymatic activity following the addition of the Ter119 scFv sequence we performed paraoxonase activity assays [27]. While all of the PON1 variants expressed by Trichoplusia ni larvae had lower catalytic efficiencies than previously reported [23] (10-25\%), the addition of the Ter119 scFv did not appear to have any significant deleterious effect on activity (Figure 3.).

\section{Ter119 scFv-PON1 in vitro binding}

To test whether the scFv-PON1 fusion proteins could bind RBCs, we incubated murine RBCs with increasing concentrations of $A B 743, A B 771$, and $A B 772$ in vitro. Using a fluorescence antibody against the $6 x$ His residue, we determined the percentage of RBCs with bound PON1 via flow cytometry. Only the AB771 fusion protein was able to significantly bind to the RBCs at $45 \mu \mathrm{g} / \mathrm{ml}$ and $75 \mu \mathrm{g} / \mathrm{ml}$ (Figure 4.). At the highest concentration tested, AB771 was on the surface of $66.56 \%$ of murine RBCs, whereas at the lowest concentration it was on the surface of over $35 \%$ of the RBCs. Both $A B 743$ and $A B 772$ were unable to bind murine RBCs in vitro.

\section{Ter119 scFv-PON1 in vivo binding}

Following the in vitro results we tested $A B 771$ in a small scale in vivo mouse study. C57BL/6 background mice were dosed at $5 \mathrm{mg} / \mathrm{kg}$ with $\mathrm{AB743}$, AB771, or PBS alone. During the experimental procedure none of the mice showed any outward appearance of illness or distress. Blood was obtained via tail snip at times $0,2,24$, and $50 \mathrm{~h}$. Using a fluorescence antibody against 6xHis, we determined the percentage of RBCs labeled with PON1 by flow cytometry. A significant treatment group by time interaction $(p<0.0001)$ was observed, 
indicating that the differences among treatment groups varied with time. AB771 was observed on the surface of a significant percentage of murine RBCs at 2 and $24 \mathrm{~h}$ after dosing (Figure 5., $p<0.0001$, two-factor ANOVA with Bonferroni post-test), and although not significant a detectable amount was seen in some mice at $50 \mathrm{~h}$. At $2 \mathrm{~h}$ after dosing, the percentage of RBC with $A B 771$ on the surface was $13.36 \%$, and over the next $48 \mathrm{~h}$ the percentage decreased to $2.35 \%$. The flow cytometry results were confirmed by measuring plasma PON1 activity (Table 1.). As expected, there was a spike in plasma PON1 activity in mice dosed with the unconjugated control (AB743) at $2 \mathrm{~h}$ after dosing. Mice administered AB771 showed no significant increased level of plasma PON1 activity when compared to control mice, and the levels were significantly lower than in AB743-treated mice at 2 and $24 \mathrm{~h}(p<0.0001$, two-factor ANOVA with Bonferroni post-test). The lack of substantial increase in plasma PON1 activity in mice treated with $A B 771$, but seen in mice dosed with unconjugated PON1, supports the flow cytometry data in suggesting that $A B 771$ can successfully bind to murine RBCs in vivo.

To summarize, all of the variants tested displayed no deleterious impact on enzymatic activity from the addition of the scFv sequence. AB771 showed an ability to bind RBCs both in vitro and in vivo as demonstrated by flow cytometry data and the lack of increased plasma PON1 activity, whereas AB772 showed no such ability. As expected, the variant $A B 743$, which lacked the Ter119 scFv sequence and therefore was unable to bind RBCs, was found to increase PON1 plasma activity but not to associate with RBCs when administered to mice.

\section{Discussion}

Desirable characteristics for any prophylactic bioscavenger include easy administration, broad specificity, lack of physiological, psychological, or immunological effects, and persistence in the circulation. For catalytic bioscavengers to reach their full potential as prophylactics against OP poisoning, increased circulatory stability is a key requirement. One strategy to increase the bioavailability of catalytic bioscavengers is to sequester them on the surface of circulating RBCs, which have a long circulatory lifespan in humans. [15]. Glycophorin A is a promising candidate for targeting prophylactics to RBCs, because of its high surface level expression [16, 17], and this strategy has been previously employed in rodent models with antiblood coagulation therapies [19, 20] by utilizing a scFv for Ter119 [22].

In this study we showed that a fusion protein of Ter119 scFv and the bioscavenger PON1 (variant VIID11) could successfully be targeted to murine RBCs. When fused to PON1, the ScFv sequence showed no significant deleterious effect on PON1 enzyme activity. One fusion protein (AB771) was able to bind a high percentage of RBCs in in vitro experiments. 

experiments. Although we do not have any results to help explain why AB771 associates with RBCs and AB772 does not, it is most likely due either to inaccessibility of the Ter119 binding site in the $A B 772$ fusion protein or to disruptions in the structure of the scFv binding site when it is located at the $\mathrm{N}$-terminus of this variant. We do not believe that the folding of AB772 has blocked the $6 \mathrm{xHis}$ tag from being recognized by our fluorescent probe, since the tag was utilized in the isolation and purification of the enzyme.

In a small scale in vivo mouse study, we were able to detect AB771 (PON1-scFv-His) on the surface of RBCs at least $24 \mathrm{~h}$ after dosing $(5 \mathrm{mg} / \mathrm{kg})$, although the percentage of labeled RBCs was much lower than in the in vitro study. The normal lifespan of a mouse RBC is around 40 days compared to 120 in humans [15, 21, 28], which, if we assume the results are scalable to humans, suggests the possibility of this strategy lasting at least 3 days in circulation. When comparing plasma PON1 activity of mice dosed with either AB771 or the unconjugated control (AB743), we observed a significant initial increase in activity in AB743 mice which decreased over time. The initial increase of paraoxonase activity was absent in AB771-treated mice. The results were interesting given the lower percentage of RBCs with $A B 771$ bound in vivo, when compared to the in vitro results, as an increase in plasma PON1 activity greater than the 2-fold increase observed could be expected. This leads to the possibility that the flow cytometry data could be underrepresenting the true amount of enzyme binding to RBCs, since it cannot account for the amount of enzyme on each positively stained cell. It is possible that when introduced via IP injection, the fusion protein binds a small subset of RBCs with a high efficiency, resulting in a lower percentage of cells decorated with a higher concentration of bioscavenger enzyme. Alternatively, it is also possible that the fusion proteins are being sequestered in the intraperitoneal space or once bound to RBCs are increasing the RBC turnover rate, leading to their more rapid clearance; these issues will be the topic of future investigation.

Previous investigators have showed near uniform labeling of RBCs by a Ter119 scFVDAF fusion protein in vivo [19], while our fusion protein was only able to label around $13 \%$ of the RBCs at the earliest time point. Since the RBC count for a normal mouse is $7-11 \times 10^{6} / \mu \mathrm{l}$ [29] labeling $13 \%$ still raises the possibility of about $1.8-2.9 \times 10^{9}$ cells carrying the bioscavenger in these mice; whether this amount of binding represents a dose of enzyme that will be protective against OP intoxication still needs to be addressed. It is also important to note that in the prior experiments, the scFv-DAF was administered via intravenous injection, while the PON1-scFv was administered via IP injection, which could account for some of the differences. Given the 
substantial decrease in PON1-scFv targeting the RBCs in vivo, when compared to in vitro, adopting a strategy similar to Zaitzev et al. [20] where RBCs are collected, loaded with the Ter119 scFv fusion protein, and then reintroduced via IV injection may ultimately be necessary to afford protection against OP agents with AB771.

Further work is still necessary to determine a prophylactically relevant binding affinity of PON1-scFv for murine RBCs and, if required, to develop strategies to improve this binding affinity. This study represents a proof of concept that a catalytically active OP bioscavenger can be successfully targeted to the surface of RBCs and remain in circulation for an extended period of time. The strategy we employed can be used to develop a prophylactic for long-term protection against $\mathrm{OP}$ nerve agent and pesticide poisoning.

\section{Acknowledgements}

This manuscript is dedicated to the memory of Dr. Gregory E. Garcia

B.J.M., C.A.H, and M.A.O were supported in part by an appointment to the Internship/Research Participation Program for the US Army Medical Research Institute of Chemical Defense, administered by the Oak Ridge Institute for Science and Education through an agreement between the US Department of Energy and the USAMRICD.

This research was supported by the Defense Threat Reduction Agency-Joint Science and Technology Office, Medical S\&T Division CBCALL12-NEURO-PRE1-2-0124

The experimental protocol was approved by the Animal Care and Use Committee at the United States Army Medical Research Institute of Chemical Defense and all procedures were conducted in accordance with the principles stated in the Guide for the Care and Use of Laboratory Animals (National Research Council, 2011), and the Animal Welfare Act of 1966 (P.L. 89-544), as amended.

The views expressed in this article are those of the authors and do not reflect the official policy of the Department of Army, Department of Defense, or the US Government. 


\section{References}

1. Sidell, F. and J. Borak, Chemical warfare agents: II. Nerve agents. Annals of Emergency Medicine, 1992. 21(7): p. 865-871.

2. Leikin, J., et al., A review of nerve agent exposure for the critical care physician. Critical Care Medicine, 2002. 30(10): p. 2346-2354.

3. CDC. Toxic Syndrome Description: Nerve Agent and Organophosphate Pesticide Poisoning 2013 Feb 142013.

4. Otto, T.C., et al., Identification and characterization of novel catalytic bioscavengers of organophosphorus nerve agents. Chemico-Biological Interactions, 2013. 203(1): p. 186-190.

5. Eddleston, M., et al., Management of acute organophosphorus pesticide poisoning. Lancet, 2008. 371(9612): p. 597-607.

6. Maynard, R. and F. Beswick, Organophosphorus compounds as chemical warfare agents, in Clincial and Experimental Toxicology of Organophosphates and Carbamates, B. Ballantyne and T.C. Marrs, Editors. 1992, Butterworth: Oxford. p. 373-385.

7. Valiyaveettil, M., et al., Protective efficacy of catalytic bioscavenger, paraoxonase 1 against sarin and soman exposure in guinea pigs. Biochemical Pharmacology, 2011. 81(6): p. 800-809.

8. Lenz, D., et al., Stoichiometric and catalytic scavengers as protection against nerve agent toxicity: A mini review. Toxicology, 2007. 233(1-3): p. 31-39.

9. Ashani, Y., et al., Stereo-specific synthesis of analogs of nerve agents and their utilization of selection and characterization of paraoxonase (PON1) catalytic scavengers. Chemico-Biological Interactions, 2010. 187(1-3): p. 362-369.

10. diTargiani, R.C., et al., In search of catalytic bioscavenger for the prophylaxis of nerve agent toxicity. Chemico-Biological Interactions, 2010. 187(1-3): p. 349-354.

11. Myers, T., et al., Characterization of human serum butyrylcholinesterase in rhesus monkeys: behavioral and physiological effects. Neurotoxicology and Teratology, 2012. 34(3): p. 323-330.

12. Genovese, R. and B. Doctor, Behavioral and pharmacological assessment of butyrylcholinesterase in rats. Pharmacology, Biochemistry, and Behavior, 1995. 51(4): p. 647654.

13. Gupta, R., et al., Directed evolution of hydrolases for prevention of G-type nerve agent intoxication. Nature Chemical Biology, 2011. 7: p. 120-125.

14. Ballas, S., Erythrocyte concentration and volume are inversely related. Clinica Chimica Acta, 1987. 164(2): p. 243-244.

15. Shemin, D. and D. Rittenberg, The life span of the human red blood cell. Journal of Biological Chemistry, 1946. 166: p. 627-636.

16. de Oliveira, S. and C. Saldanha, An overview about erythrocyte membrane. Clinical Hemorheology and Microcirculation, 2010. 44: p. 63-74.

17. Poole, J., Red cell antigens on band3 and glycophorin A. Blood Reviews, 2000. 14(1): p. 31-43.

18. Gahmber, C., et al., Biosynthesis of the major human red cell sialoglycoprotein, glycophorin A: A review. Revue Française de Transfusion et Immuno-hématologie, 1981. 24(1): p. 53-73.

19. Spitzer, D., et al., ScFv-mediated in vivo targeting of DAF to erythrocytes inhibits lysis by complement. Molecular Immunology, 2003. 40: p. 911-919.

20. Zaitzev, S., et al., Targeting of a mutant plasminogen activator to circulating red blood cells for prophylactic fibrinolysis. The Journal of Pharmacology and Experimental Therapeutics, 2010. 332(3): p. 1022-1031.

21. Magnani, M., et al., Effect of age on some properties of mice erythrocytes. Mechanisms of ageing and development, 1988. 42(1): p. 37-47. 
22. Kina, T., et al., The monoclonal antiboy Ter-119 recognizes a molecule associated with glycophorin A and specifically marks the late stages of murine erythroid lineage. British Journal of Haematology, 2000. 109(2): p. 280-287.

23. Mata, D.G., et al., Investigation of evolved paraoxonase-1 variants for prevention of organophosphorouss pesticide compound intoxication. Journal of Pharmacology and Experimental Therapeutics, 2014. 349(3): p. 549-558.

24. Goldsmith, M., et al., Evolved stereoselective hydrolases for broad-spectrum G-type nerve agent detoxificatoin. Chemistry and Biology, 2012. 19(4): p. 456-466.

25. Kovaleva, E., et al., Recombinant protein production in insect larvae: host choice, tissue distribution, and heterologous gene instability. Biotechnology Letters, 2009. 31(3): p. 381-386.

26. Schmitt, J., H. Hess, and H.G. Stunnenberg, Affinity purification of histidine-tagged proteins. Molecular Biology Reports, 1993. 18(3): p. 223-230.

27. Yeung, D., D. Lenz, and D.M. Cerasoli, Analysis of active-site amino-acid residues of human serum paraoxonase using competitive substrates. The FEBS Journal, 2005. 272(9): p. 2225-2230. 


\section{AB771}

\begin{tabular}{|c|c|c|c|}
\hline PON1 (VIID11) & $\begin{array}{c}\text { GSSSSGSSSS } \\
\text { linker }\end{array}$ & Ter119 scFv & His6x \\
\hline
\end{tabular}

\section{AB772}

\begin{tabular}{|c|c|c|c|}
\hline Ter119 scFv & $\begin{array}{c}\text { GSSSSGSSSS } \\
\text { linker }\end{array}$ & PON1 (VIID11) & His6x \\
\hline
\end{tabular}

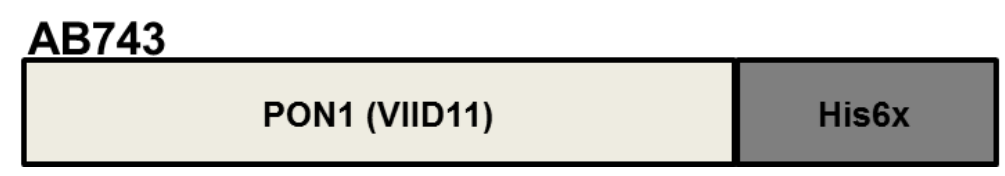

Figure 1. Schematic of Fusion Protein Construction. The design strategy for scFv-PON1 fusion proteins. The sequence for the VIID11 variant of PON1 was linked to the Ter119 scFv sequence by a GSSSSGSSSS sequence. A C terminal His6x residue was added to aid in isolation and purification. 


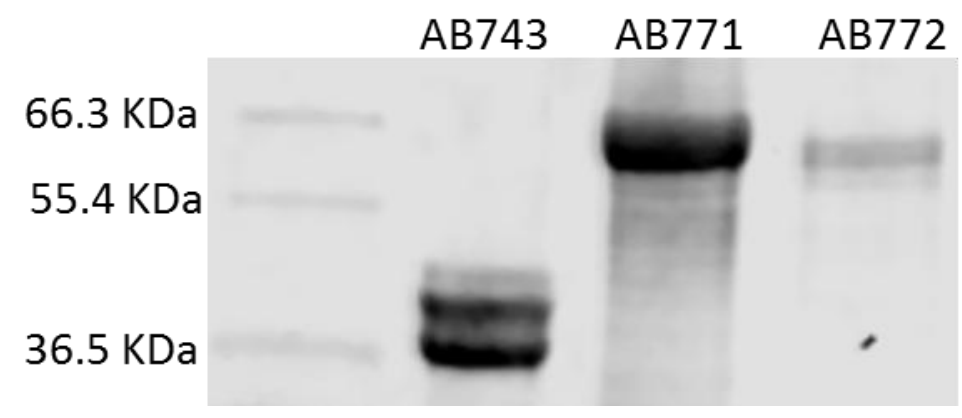

Figure 2. SDS-PAGE of fusion proteins. An SDS-PAGE was run on fusion proteins obtained from Chesapeake PERL to confirm their identities. All proteins ran at the sizes expected, indicating that $A B 771$ and $A B 772$ are the fusion proteins of scFv-PON and that $A B 743$ is the unconjugated PON control. 


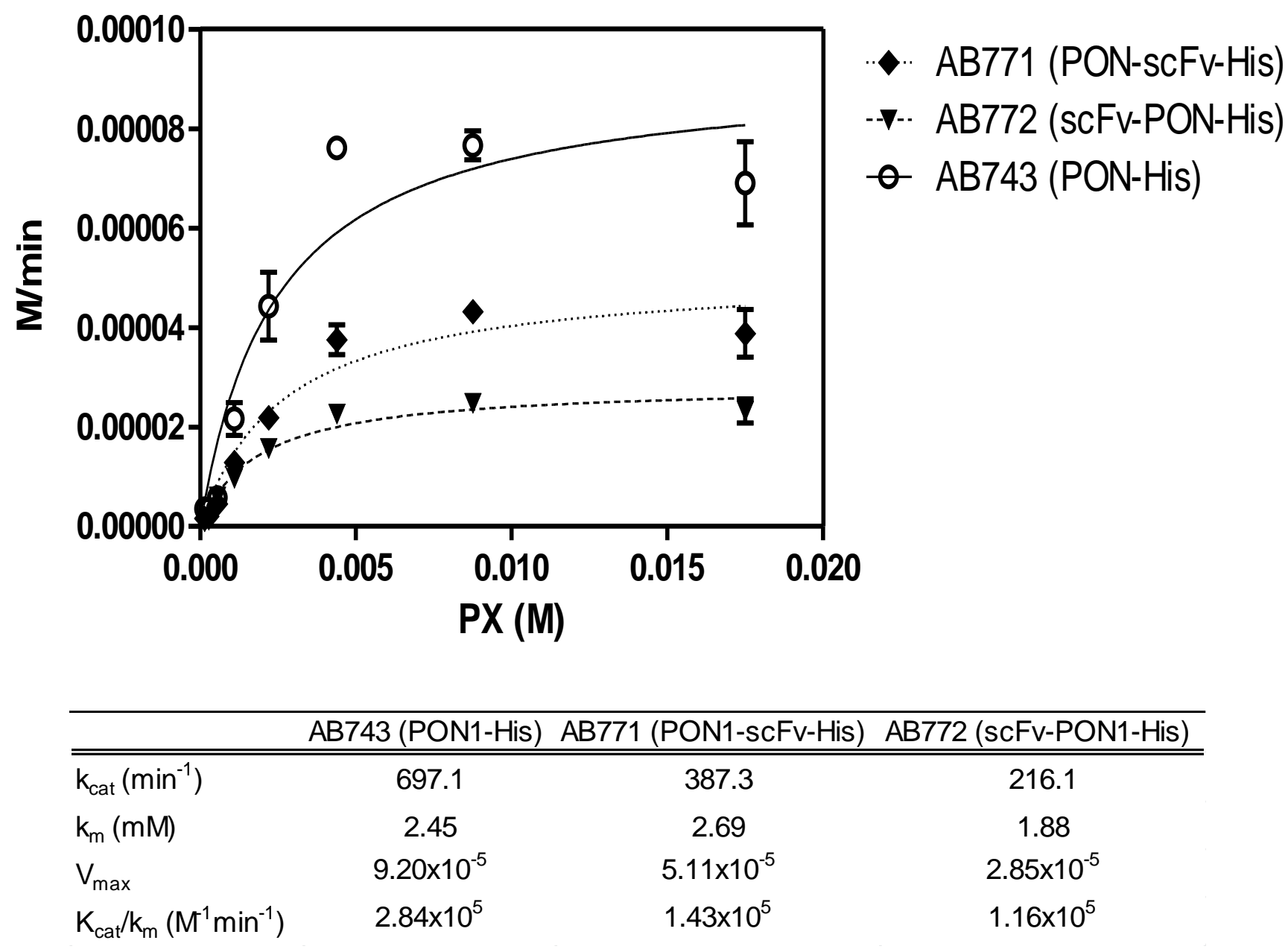

Figure 3. In vitro Paraoxonase activity. Activity of the three fusion proteins was tested against paraoxon. The assay was run in a 96-well plate format with a final reaction volume of $200 \mu \mathrm{l}$ per well. PON1 fusion proteins were added at a volume of $10 \mu \mathrm{l}$ to the plates (final concentration of $0.13 \mu \mathrm{M}$ ), along with $180 \mu \mathrm{l}$ of assay buffer consisting of $50 \mathrm{mM} \mathrm{HEPES} \mathrm{pH} 7.2$ and $10 \mathrm{mM} \mathrm{CaCl}$. Increasing concentrations of paraoxon were added at volumes of $10 \mu \mathrm{l}$ to each well, and plates were read in kinetic mode at a wavelength of $412 \mathrm{~nm}$, and read at $20 \mathrm{sec}$ intervals for $10 \mathrm{~min}$. The reaction rate was converted from $\mathrm{mAU} / \mathrm{min}$ to $\mathrm{M} / \mathrm{min}$ and plotted vs. paraoxon concentration. $V_{\max }$ and $\mathrm{k}_{\mathrm{m}}$ were calculated from the plot using the Michaelis-Menton model, and $\mathrm{k}_{\text {cat }}$ and the catalytic efficiency $\left(\mathrm{k}_{\mathrm{ca}} / \mathrm{k}_{\mathrm{m}}\right)$ were calculated from those values. $n=3$, Error Bars $=$ Standard Deviation . 
Figure 4. In vitro binding of scFv-PON variants to RBCs. RBCs were incubated with scFvPON variants and then with a fluorescent antibody for $6 x$ His. Cells were analyzed by flow cytometry to determine the percentage of cells labeled with fusion protein. There was no detectable binding of $A B 743$ (unconjugated PON1) or AB772 (scFv-PON1) at any of the concentrations tested. AB771 (PON1-scFv) had significant binding at $45 \mu \mathrm{g} / \mathrm{ml}$ and $75 \mu \mathrm{g} / \mathrm{ml}$ when compared to the unconjugated PON variant. The results indicate that $A B 771$ can successfully bind to the surface of RBCs. ${ }^{*} p<0.017$, Student's T-test vs. AB743 with Bonferroni correction. $n=3$, Error Bars $=$ Standard Deviation. 
Figure 5. In vivo binding of $\mathrm{AB} 771$ to RBCs. C57BL/6 background mice were dosed with 5 $\mathrm{mg} / \mathrm{kg}$ of PON1-scFV (AB771), unconjugated PON1 (AB743), or PBS only. Blood samples were obtained at 2, 24, and $50 \mathrm{~h}$, and the percentage of RBCs labeled with enzyme was determined by flow cytometry using a fluorescent antibody for His6x. The values for individual mice are represented by open circles (AB771), gray circles (AB743), and closed squares (PBS). Average values for each group are represented by a line. Background fluorescence was measured on $0.15 \% \pm 0.06 \%$ of red blood cells. A two-factor ANOVA with repeated measures was used to compare the three treatment groups and three observation times. A significant treatment group by time interaction $(p<0.0001)$ was observed, indicating that the differences among treatment groups varied with time. Therefore, a Bonferroni post-test was used to compare pairs of treatments at each time point. ${ }^{*} p<0.0001$ vs. PBS; ${ }^{\wedge} p<0.0001$ vs. AB743; PBS and $\mathrm{AB} 743 n=5, \mathrm{AB} 771 n=9$ 
Table 1. Normalized plasma PON1 activity

\begin{tabular}{rccc}
\hline & PBS & AB771 & AB743 \\
\hline \hline $2 \mathrm{~h}$ & $1.08 \pm 0.19$ & $2.48 \pm 0.80^{\wedge}$ & $22.91 \pm 5.33^{*}$ \\
$24 \mathrm{~h}$ & $1.26 \pm 0.34$ & $1.96 \pm 0.61^{\wedge}$ & $7.12 \pm 1.50^{*}$ \\
$50 \mathrm{~h}$ & $1.03 \pm 0.26$ & $1.50 \pm 0.46$ & $2.24 \pm 0.51$
\end{tabular}

PON1 activity was normalized to baseline at time 0 for each mouse (1.00), \pm standard deviation. A two-factor ANOVA with repeated measures was used to compare the three treatment groups and three observation times. A significant treatment group by time interaction $(p<0.0001)$ was observed, indicating that the differences among treatment groups varied with time. Therefore, a Bonferroni post-test was used to compare pairs of treatments at each time point. ${ }^{*} p<0.0001$ vs. PBS, ${ }^{\wedge} p<0.0001$ vs. AB743, $n=4-9$ 
Table 2. Normalized plasma PON1 activity

\begin{tabular}{cccc}
\hline & PBS & AB771 & AB743 \\
\hline \hline $2 \mathrm{hrs}$ & $1.08 \pm 0.19$ & $2.61 \pm 0.74^{* \wedge}$ & $22.91 \pm 5.33^{*}$ \\
$24 \mathrm{hr}$ & $1.26 \pm 0.34$ & $2.01 \pm 0.63^{* \wedge}$ & $7.12 \pm 1.50^{*}$ \\
$50 \mathrm{hrs}$ & $1.03 \pm 0.26$ & $1.62 \pm 0.32^{*}$ & $2.24 \pm 0.51^{*}$
\end{tabular}

PON1 activity was normalized to baseline at time 0 for each mouse (1.00), \pm Standard

Deviation, ${ }^{*} p<0.0167$ Student's T Test with Bonferroni correction vs. PBS, ${ }^{\wedge} p<0.001$

Student's T Test with Bonferroni correction vs. AB743, $n=5-9$ 